\title{
A Bayesian Estimation and Prediction of Gompertz Extension Distribution Using the MCMC Method
}

\author{
Arun Kumar Chaudhary ${ }^{1 *}$ and Vijay Kumar ${ }^{2}$ \\ ${ }^{1}$ Faculty of Management Science, Nepal Commerce Campus, \\ Tribhuwan University, Nepal \\ ${ }^{2}$ Department of Mathematics and Statistics, DDU Gorakhpur University, Uttar \\ Pradesh, India \\ *Corresponding Author \\ akchaudhary1@yahoo.com
}

\begin{abstract}
In this paper, the Markov chain Monte Carlo (MCMC) method is used to estimate the parameters of the Gompertz extension distribution based on a complete sample. We have developed a procedure to obtain Bayes estimates of the parameters of the Gompertz extension distribution using Markov Chain Monte Carlo (MCMC) simulation method in OpenBUGS, established software for Bayesian analysis using Markov Chain Monte Carlo (MCMC) methods. We have obtained the Bayes estimates of the parameters, hazard and reliability functions, and their probability intervals are also presented. We have applied the predictive check method to discuss the issue of model compatibility. A real data set is considered for illustration under uniform and gamma priors
\end{abstract}

Keywords

Bayesian estimation, Maximum likelihood estimation, markov chain monte carlo, model validation, OpenBUGS.

\section{Introduction}

The cumulative distribution function (CDF) of the Gompertz distribution (GZ), Gompertz (1825) is given by

$F_{G Z}(x ; \alpha, \lambda)=1-\exp \left\{-\lambda\left(e^{\alpha x}-1\right)\right\} ; \quad \alpha, \lambda>0 ; \mathrm{x} \geq 0$ The corresponding hazard rate function is given by $h_{G Z}(x ; \alpha, \lambda)=\alpha \lambda e^{\alpha x} ; \quad \alpha, \lambda>0 ; \mathrm{x} \geq 0$.

Adding one or more parameters to distribution makes it more productive and more flexible for modelling data. There are different ways for adding parameter(s) to a distribution. Such addition of parameters makes the resulting distribution richer and more flexible for modelling data. Marshall and Olkin $(1997,2007)$, added one positive parameter to a given (general) survival function.

The Gompertz distribution with power parameter (GP), Marshall and Olkin (2007) has cdf
$F_{G P}(x ; \lambda, \xi, \alpha)=1-\exp \left\{-\xi\left(e^{(\lambda x)^{\alpha}}-1\right)\right\}$

hazard rate

$h_{G P}(x ; \lambda, \xi, \alpha)=\xi \lambda \alpha(\lambda x)^{\alpha-1} e^{(\lambda x)^{\alpha}} ; \lambda, \xi, \alpha>0 ; \mathrm{x} \geq 0$

and density

$f_{G P}(x ; \lambda, \xi, \alpha)=\xi \lambda \alpha(\lambda x)^{\alpha-1} e^{(\lambda x)^{\alpha}} \exp \left\{-\xi\left(e^{(\lambda x)^{\alpha}}-1\right)\right\}$

With the frailty parameter $\xi=1$, this distribution is discussed by Dhillon (1981), Leemis (1986) and Kunitz (1989). With $\lambda=1$, the distribution is proposed by Chen (2000) the general case has been called a "modified Weibull extension" and is studied by Xie et al., (2002), as well as by Murthy, et al., (2004). The various authors note that the hazard rate of this distribution is bathtub shaped when $\alpha<1$. When $\alpha \geq 1$, the hazard rate is increasing. The hazard rate is convex for all $\alpha>0$. 
The Gompertz distribution has a limiting exponential distribution. Similarly, the Gompertz distribution with power parameter has a limiting Weibull distribution. Because of this convergence, the Gompertz distribution with power parameter is called a "Weibull extension" by Murthy et al., (2004).

Another modified version of Gompertz distribution named as the Gompertz distribution with hazard power parameter has been presented in Marshall and Olkin (2007). We prefer to call the Gompertz distribution with hazard power parameter as the "Gompertz Extension" distribution. Note that the Gompertz distribution is a particular member of the "Gompertz Extension" distribution. We do not find any systematic study classical as well as Bayesian on Gompertz Extension (GZE) distribution in the literature.

\section{Gompertz Extension (GZE) \\ Distribution: model analysis}

\section{Cumulative distribution function (CDF):}

The distribution function of Gompertz extension distribution with three parameters is given by

$$
F(x ; \alpha, \lambda, \theta)=1-\exp \left\{-\lambda\left(e^{\alpha x}-1\right)^{\theta}\right\}
$$

where $\alpha, \lambda$ and $\theta$ are the parameters. The Gompertz extension distribution will be denoted by $\operatorname{GZE}(\alpha, \lambda, \theta)$

\section{Probability density function (PDF):}

The probability density function is given by

$$
f(x ; \alpha, \lambda, \theta)=\alpha \lambda \theta e^{\alpha x}\left(e^{\alpha x}-1\right)^{\theta-1} \exp \left\{-\lambda\left(e^{\alpha x}-1\right)^{\theta}\right\}
$$

Some of the typical GZE density functions for different values of $\theta$ and $(\alpha=1, \lambda=1)$ are depicted in Figure 2.1. It is clear from Figure 2.1 that the density function of the GZE distribution can take different shapes.

\section{The reliability/survival function (SF):}

The reliability/survival function is

$$
R(x ; \alpha, \lambda, \theta)=\exp \left\{-\lambda\left(e^{\alpha x}-1\right)^{\theta}\right\} ; \quad \alpha, \lambda, \theta>0 ; \mathrm{x} \geq 0
$$

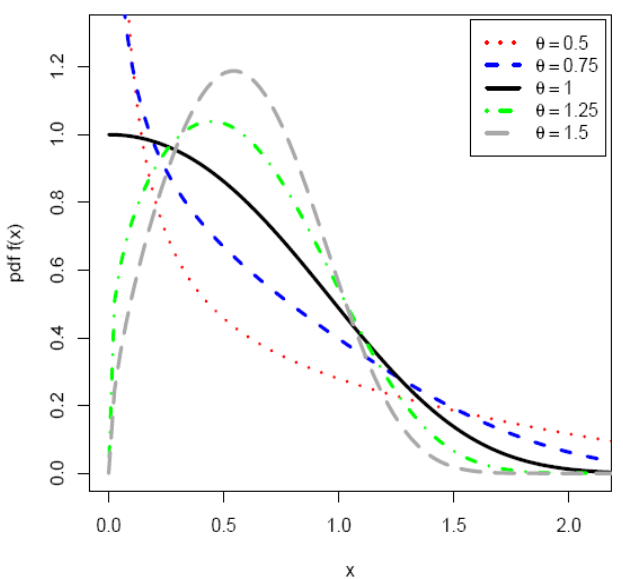

Fig 2.1 The PDF of the GZE for $\alpha=1, \lambda=1$ and different values of $\theta$.

\section{The hazard rate function (HRF):}

The hazard rate function is

$$
h(x ; \alpha, \lambda, \theta)=\alpha \lambda \theta e^{\alpha x}\left(e^{\alpha x}-1\right)^{\theta-1} ; \quad \alpha, \lambda, \theta>0 ; \mathrm{x} \geq 0
$$

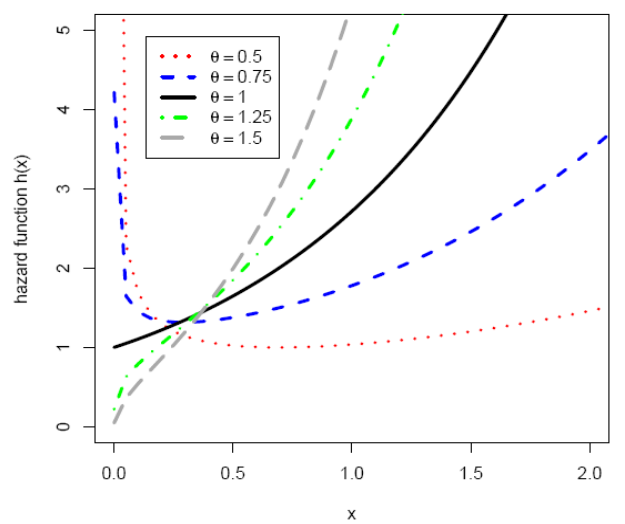

Fig 2.2: The HRF of GZE distribution for $\alpha=1$, $\lambda=1$ and different values of $\theta$.

It is increasing when $\theta \geq 1$ and when $\theta<1$ the hazard rate has a minimum at $\mathrm{x}=-(\log \theta / \alpha)$. Figure 2.2 exhibits the different hazard rate functions for GZE distribution. 


\section{The cumulative hazard function (CHF) and failure rate average (FRA):}

The cumulative hazard function $H(x)$ defined as

$$
H(x)=-\log \{1-F(x)\}
$$

Another relevant function useful in reliability analysis is failure rate average (FRA). The failure rate average of $\mathrm{X}$ is given by

$$
\operatorname{FR} A(x)=\frac{H(x)}{x}=\frac{1}{x} \int_{0}^{x} h(x) d x(2.6)
$$

where $H(x)$ is the cumulative hazard function. An analysis for $F R A(x)$ on $\mathrm{x}$ permits to obtain the IFRA and DFRA classes.

\section{The quantile function $(Q F)$ :}

The quantile function is given by

$$
x_{p}=\frac{1}{\alpha} \ln \left[1+\left\{-\frac{1}{\lambda} \log (1-p)\right\}^{1 / \theta}\right] ; 0<p<1
$$

\section{The Random Deviate Generation:}

The random deviate can be generated from $\operatorname{GZE}(\alpha, \lambda, \theta)$ by

$$
x=\frac{1}{\alpha} \ln \left[1+\left\{-\frac{1}{\lambda} \log (1-u)\right\}^{1 / \theta}\right] ; 0<u<1
$$

where $u$ has the $U(0,1)$ distribution. For model choice based on information criterion, the values of AIC and BIC can be used.

\section{Maximum likelihood estimation (MLE) and information matrix}

In this section, we briefly discuss the maximum likelihood estimators (MLE's) of the Gompertz extension distribution and discuss their asymptotic properties to obtain approximate confidence intervals based on MLE's.

Let $x=\left(x_{1}, \ldots, x_{n}\right)$ be a random sample of size $n$ from $\operatorname{GZE}(\alpha, \lambda, \theta)$, and then the log-likelihood function $\ell(\alpha, \lambda, \theta \mid \underline{x})$ can be written as;

$$
\begin{gathered}
\ell(\alpha, \lambda, \theta \mid \underline{x})=n \log \alpha+n \log \lambda+n \log \theta+(\theta-1) \sum_{i=1}^{n} \log \left(e^{\alpha x_{i}}-1\right) \\
+\alpha \sum_{i=1}^{n} x_{i}-\lambda \sum_{i=1}^{n}\left(e^{\alpha x_{i}}-1\right)^{\theta}
\end{gathered}
$$

Therefore, to obtain the MLE's of $\alpha, \lambda$ and $\theta$, we can maximize (3.1) directly with respect to $\alpha, \lambda$ and $\theta$ or we can solve the following two non-linear equations using the Newton-Raphson method :

$$
\begin{aligned}
& \frac{\partial \ell}{\partial \alpha}=\frac{n}{\alpha}+\sum_{i=1}^{n} x_{i}+(\theta-1) \sum_{i=1}^{n} \frac{x_{i} e^{\alpha x_{i}}}{\left(e^{\alpha x_{i}}-1\right)}-\lambda \theta \sum_{i=1}^{n} x_{i} e^{\alpha x_{i}}\left(e^{\alpha x_{i}}-1\right)^{\theta-1}=0 \\
& \frac{\partial \ell}{\partial \lambda}=\frac{n}{\lambda}-\sum_{i=1}^{n}\left(e^{\alpha x_{i}}-1\right)^{\theta}=0
\end{aligned}
$$

and

$$
\frac{\partial \ell}{\partial \theta}=\frac{n}{\theta}+\sum_{i=1}^{n} \log \left(e^{\alpha x_{i}}-1\right)-\lambda \sum_{i=1}^{n}\left(e^{\alpha x_{i}}-1\right)^{\theta} \log \left(e^{\alpha x_{i}}-1\right)=0 .
$$

Let us denote the parameter vector by $\underline{\delta}=(\alpha, \lambda, \theta)$ and the corresponding MLE of $\underline{\delta}$ as $\underline{\hat{\delta}}=(\hat{\alpha}, \hat{\lambda}, \hat{\theta})$ then the asymptotic normality results in

$$
(\underline{\hat{\delta}}-\underline{\delta}) \rightarrow N_{3}\left(0,(I(\underline{\delta}))^{-1}\right)(3.2
$$

where $\mathrm{I}(\underline{\delta})$ is the Fisher's information matrix given by 


$$
I(\underline{\delta})=-\left(\begin{array}{ccc}
E\left(\frac{\partial^{2} \ell}{\partial \alpha^{2}}\right) & E\left(\frac{\partial^{2} \ell}{\partial \alpha \partial \lambda}\right) & E\left(\frac{\partial^{2} \ell}{\partial \alpha \partial \theta}\right) \\
E\left(\frac{\partial^{2} \ell}{\partial \lambda \partial \alpha}\right) & E\left(\frac{\partial^{2} \ell}{\partial \lambda^{2}}\right) & E\left(\frac{\partial^{2} \ell}{\partial \lambda \partial \theta}\right) \\
E\left(\frac{\partial^{2} \ell}{\partial \theta \partial \alpha}\right) & E\left(\frac{\partial^{2} \ell}{\partial \theta \partial \lambda}\right) & E\left(\frac{\partial^{2} \ell}{\partial \theta^{2}}\right)
\end{array}\right)
$$

In practice, it is useless that the MLE has asymptotic variance $(I(\underline{\delta}))^{-1}$ because we do not know $\underline{\delta}$. Hence, we approximate the asymptotic variance by "plugging in" the estimated value of the parameters. The standard procedure is to use observed Fisher information matrix $O(\underline{\hat{\delta}})$ (as an estimate of the information matrix $\mathrm{I}(\delta))$ given by

$$
O(\underline{\hat{\delta}})=-\left(\begin{array}{ccc}
\frac{\partial^{2} \ell}{\partial \alpha^{2}} & \frac{\partial^{2} \ell}{\partial \alpha \partial \lambda} & \frac{\partial^{2} \ell}{\partial \alpha \partial \theta} \\
\frac{\partial^{2} \ell}{\partial \lambda \partial \alpha} & \frac{\partial^{2} \ell}{\partial \lambda^{2}} & \frac{\partial^{2} \ell}{\partial \lambda \partial \theta} \\
\frac{\partial^{2} \ell}{\partial \theta \partial \alpha} & \frac{\partial^{2} \ell}{\partial \theta \partial \lambda} & \frac{\partial^{2} \ell}{\partial \theta^{2}}
\end{array}\right)_{\mid(\hat{\alpha}, \hat{\lambda}, \hat{\theta})}=-\left.H(\underline{\delta})\right|_{\underline{\delta}=\underline{\hat{\delta}}}(3
$$

where $\mathrm{H}$ is the Hessian matrix, $\underline{\delta}=(\alpha, \lambda, \theta)$ and $\underline{\hat{\delta}}=(\hat{\alpha}, \hat{\lambda}, \hat{\theta})$. The Newton-Raphson algorithm to maximize the likelihood produces the observed information matrix. Therefore, the variance-covariance matrix is given by

$$
\left(-\left.H(\underline{\delta})\right|_{\underline{\delta}=\underline{\hat{\delta}}}\right)^{-1}=\left(\begin{array}{ccc}
\operatorname{var}(\hat{\alpha}) & \operatorname{cov}(\hat{\alpha}, \hat{\lambda}) & \operatorname{cov}(\hat{\alpha}, \hat{\theta}) \\
\operatorname{cov}(\hat{\lambda}, \hat{\alpha}) & \operatorname{var}(\hat{\lambda}) & \operatorname{cov}(\hat{\lambda}, \hat{\theta}) \\
\operatorname{cov}(\hat{\theta}, \hat{\alpha}) & \operatorname{cov}(\hat{\theta}, \hat{\lambda}) & \operatorname{var}(\hat{\theta})
\end{array}\right)(3.5)
$$

Hence, from the asymptotic normality of MLEs, approximate $100(1-\gamma) \%$ confidence intervals for $\alpha, \lambda$ and $\theta$ can be constructed as

$$
\hat{\alpha} \pm z_{\gamma / 2} \sqrt{\operatorname{var}(\hat{\alpha})} \hat{\lambda} \pm z_{\gamma / 2} \sqrt{\operatorname{var}(\hat{\lambda})} \text { and } \hat{\theta} \pm z_{\gamma / 2} \sqrt{\operatorname{var}(\hat{\theta})}(3.6)
$$

where $z_{\gamma / 2}$ is the upper percentile of standard normal variate.

\section{Bayesian Model Formulation}

The Bayesian model is constructed by specifying the prior distributions for the model parameters $\alpha, \lambda$ and $\theta$, and then multiplying with the likelihood function to obtain the posterior distribution function.

- Probability Model: $f(x \mid \alpha, \lambda, \theta)$

- Prior distribution: $p(\alpha, \lambda, \theta)$

- Data: $\underline{x}=\left(x_{1}, \ldots, x_{n}\right)$

Given a set of data $\underline{x}=\left(x_{1}, \ldots, x_{n}\right)$, the likelihood function is

$L(\alpha, \lambda, \theta \mid \underline{x})=\alpha^{n} \lambda^{n} \theta^{n}\left\{\prod_{i=1}^{n}\left(e^{\alpha x_{i}}-1\right)^{\theta-1}\right\} \exp \left\{\alpha \sum_{i=1}^{n} x_{i}-\lambda \sum_{i=1}^{n}\left(e^{\alpha x_{i}}-1\right)^{\theta}\right\}$ 
Denote the prior distributions of $\alpha, \lambda$ and $\theta p(\alpha, \lambda, \theta)$ as. The joint posterior is $p(\alpha, \lambda, \theta \mid \underline{x}) \propto L(\alpha, \lambda, \theta \mid \underline{x}) p(\alpha, \lambda, \theta)$

\section{Prior Distributions:}

We assume the independent uniform for $\alpha \sim U\left(a_{1}, b_{1}\right)$ and gamma priors for $\lambda \sim G\left(a_{2}, b_{2}\right)$ and $\theta \sim G\left(a_{3}, b_{3}\right)$ as

$$
\begin{aligned}
& p(\alpha)=\frac{1}{b_{1}-a_{1}} ; a_{1}<\alpha<b_{1} \\
& p(\lambda)=\frac{b_{2}^{a_{2}}}{\Gamma\left(a_{2}\right)} \lambda^{a_{2}-1} \mathrm{e}^{-b_{2} \lambda} ; \lambda>0,\left(a_{2}, b_{2}\right)>0
\end{aligned}
$$

and

$$
p(\theta)=\frac{b_{3}^{a_{3}}}{\Gamma\left(a_{3}\right)} \theta^{a_{3}-1} \mathrm{e}^{-b_{3} \theta} ; \theta>0,\left(a_{3}, b_{3}\right)>0
$$

\section{Posterior Distribution:}

Combining the likelihood function with the prior via Bayes' theorem yields the posterior up to proportionality as

$$
\begin{gathered}
p(\alpha, \lambda, \theta \mid \underline{x})=\alpha^{n} \lambda^{n} \theta^{n}\left\{\prod_{i=1}^{n}\left(e^{\alpha x_{i}}-1\right)^{\theta-1}\right\} \exp \left\{\alpha \sum_{i=1}^{n} x_{i}-\lambda \sum_{i=1}^{n}\left(e^{\alpha x_{i}}-1\right)^{\theta}\right\} \\
\frac{1}{b_{1}-a_{1}} \frac{b_{2}{ }^{a_{2}}}{\Gamma a_{2}} e^{-b_{2} \lambda} \lambda^{a_{2}-1} \frac{b_{3}{ }^{a_{3}}}{\Gamma a_{3}} e^{-b_{3} \theta} \theta^{a_{3}-1} \\
p(\alpha, \lambda, \theta \mid \underline{x}) \propto \alpha^{n} \lambda^{n+a_{2}-1} \theta^{n+a_{3}-1}\left\{\prod_{i=1}^{n}\left(e^{\alpha x_{i}}-1\right)^{\theta-1}\right\} \\
\exp \left\{-\lambda \sum_{i=1}^{n}\left(e^{\alpha x_{i}}-1\right)^{\theta}-b_{2} \lambda-b_{3} \theta\right\}
\end{gathered}
$$

The posterior is complicated, and no close form inferences appear possible. We, therefore, propose to consider MCMC methods to simulate samples from the posterior so that sample-based inferences can be quickly drawn. Markov chain Monte Carlo draws samples by running a cleverly constructed Markov chain that eventually converges to the target distribution (called stationary or equilibrium) which, in our case, is the posterior distribution $p(\alpha, \lambda, \theta \mid \underline{x})$. There are many ways of constructing these chains, but all of them, including the Gibbs sampler, Geman and Geman (1984); Gelfand and Smith (1990) are special cases of the general framework of Metropolis, et al., (1953) and Hastings (1970). Recently, Afify et al., (2019) discussed the Bayesian estimation of three parameter exponential distribution and Alizadeh et al. (2020) presented the estimation procedures for odd log-logistic Lindley-G family of distributions.

\section{Gibbs sampler: algorithm}

For Gibbs sampler implementation, the full conditionals for $\alpha, \lambda$ and $\theta$ up to proportionality can be specified as

(i) The full conditional distribution of the parameter $\alpha$ for given $\lambda, \theta$ and $\underline{x}$

$$
p(\alpha \mid \lambda, \theta, \underline{x}) \propto \alpha^{n}\left\{\prod_{i=1}^{n}\left(e^{\alpha x_{i}}-1\right)^{\theta-1}\right\} \exp \left\{-\lambda \sum_{i=1}^{n}\left(e^{\alpha x_{i}}-1\right)^{\theta}\right\}
$$


(ii) The full conditional distribution of the parameter $\lambda$ for given $\alpha, \theta$ and $\underline{x}$

$$
p(\lambda \mid \alpha, \theta, \underline{x}) \propto \lambda^{n+a_{2}-1} \exp \left[-\lambda\left\{\sum_{i=1}^{n}\left(e^{\alpha x_{i}}-1\right)^{\theta}+b_{2}\right\}\right]
$$

(iii) The full conditional distribution of the parameter $\theta$ for given $\alpha, \lambda$ and $\underline{x}$

$$
p(\theta \mid \alpha, \lambda, \underline{x}) \propto \theta^{n+a_{3}-1}\left\{\prod_{i=1}^{n}\left(e^{\alpha x_{i}}-1\right)^{\theta-1}\right\} \exp \left\{-\lambda \sum_{i=1}^{n}\left(e^{\alpha x_{i}}-1\right)^{\theta}-b_{3} \theta\right\}
$$

We shall use OpenBUGS software to obtain posterior samples. As the Gompertz extension distribution is not available in OpenBUGS, it requires the incorporation of a module in ReliaBUGSsubsystem of OpenBUGS for Gompertz extension (Kumar, et al., 2010; Lunn 2010).

A module dgpz.ext_T (alpha, lambda, theta) is written in Component Pascal for Gompertz extension to perform full Bayesian analysis in OpenBUGS using the method described in Thomas et al. (2006); Thomas (2010); Kumar, et al., (2010); Lunn, et al., (2010); Lunn (2013). It is important to note that this module can be used for any set of suitable priors of the model parameters. Almost all aspects of the model in Bayesian framework can be studied using the developed module dgpz.ext_T (alpha, lambda, theta)

\section{Gibbs Sampler: Implementation}

1. Select an initial value $\underline{\delta}^{(0)}=\left(\alpha^{(0)}, \lambda^{(0)}, \theta^{(0)}\right)$ to start the chain.

2. Suppose at the ith-step, $\underline{\delta}=(\alpha, \lambda, \theta)$ takes the value $\underline{\delta}^{(i)}=\left(\alpha^{(i)}, \lambda^{(i)}, \theta^{(i)}\right)$ than from full conditionals, we generate.

$$
\begin{aligned}
& \alpha^{(i+1)} \text { from } p\left(\alpha \mid, \lambda^{(i)}, \theta^{(i)}, \underline{x}\right) \\
& \lambda^{(i+1)} \text { from } p\left(\lambda \mid, \alpha^{(i+1)}, \theta^{(i)}, \underline{x}\right) \text { and } \\
& \theta^{(i+1)} \text { from } p\left(\theta \mid \alpha^{(i+1)}, \lambda^{(i+1)}, \underline{x}\right) .
\end{aligned}
$$

3. This completes a transition from $\underline{\delta}^{(i)}$ to $\underline{\delta}^{(i+1)}$

4. Repeat step 2, $N$ times.

\section{MCMC Output: Posterior Sample}

Monitor the convergence using convergence diagnostics (trace and ergodic mean plots). Suppose that convergence has been reached after ' $B$ ' iterations (the burn-in period). Discard the observations $\left(\underline{\delta}^{(1)}, \underline{\delta}^{(2)}, \ldots, \underline{\delta}^{(B)}\right)$ and retain the observations

$$
\left(\underline{\delta}^{(\mathrm{B}+1+(\mathrm{j}-1) \mathrm{L})} ; \mathrm{B}+1+(\mathrm{M}-1) \mathrm{L} \leq \mathrm{N} ; \mathrm{j}=1,2, \ldots, \mathrm{M} ; \mathrm{L} \geq 1\right)
$$

Which are viewed as being an independent sample from the stationary distribution of the Markov chain that is typically the posterior distribution, where $L$ is the lag (or thin interval).

Consider $\left(\underline{\delta}^{(1)}, \ldots, \underline{\delta}^{(j)}, \ldots, \underline{\delta}^{(M)}\right)$ as the MCMC output (posterior sample) for the posterior analysis

$$
\underline{\delta}^{(j)}=\left(\alpha^{(j)}, \lambda^{(j)}, \theta^{(j)}\right) ; j=1,2, \ldots, M .
$$

Thus, MCMC output is referred to as the sample after removing the initial iterations (produced during the burn-in period) and considering the appropriate lag.

The Bayes estimates $\underline{\delta}=(\alpha, \lambda, \theta)$ are given by

$$
\hat{\alpha}=\frac{1}{M} \sum_{j=1}^{M} \alpha^{(j)} ; \hat{\lambda}=\frac{1}{M} \sum_{j=1}^{M} \lambda^{(j)} ; \hat{\theta}=\frac{1}{M} \sum_{j=1}^{M} \theta^{(j)}
$$

\section{Data analysis}

Data Set: The real data set is considered for illustration of the proposed methodology. The data gives 100 observations on breaking stress of carbon fibres (in Gba), (Nichols and Padgett 2006).

\section{Exploratory data analysis (EDA)}

The goal of data analysis is to gain information from the data. The modern statistical data analysis tools include exploratory data analysis. 
Table 5.1 Summary Statistics

\begin{tabular}{cccccccc}
\hline Min. & $\mathbf{Q}_{1}$ & Median & Mean & $\mathbf{Q}_{3}$ & Max. & Skewness & Kurtosis \\
\hline 0.390 & 1.840 & 2.700 & 2.621 & 3.220 & 5.560 & 0.3626 & 0.0432 \\
\hline
\end{tabular}
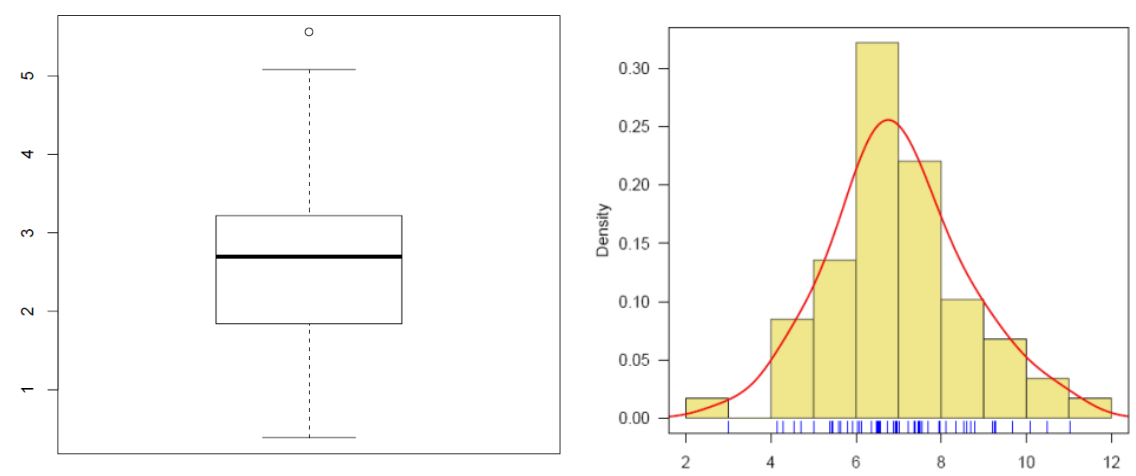

Fig 5.1 The boxplot (left panel), histogram and density plot (right panel) along with the data points.

Exploratory data analysis is a set of methods to display and summarize the data:

- displaying the data in a graph that shows overall patterns and unusual observations (bar chart, histogram, density curve)

- computing descriptive statistics that summarize specific aspects of the data (centre and spread).

We have applied the basic EDA techniques, and the results are presented in Table 5.1, and corresponding graphs are displayed in Figure 5.1. The total-time-on-test (TTT) plot is a graphical procedure to get some idea about the shape of the hazard function. We have used the empirical version of the scaled TTT plot (Aarset 1987). We have plotted the empirical version of the scaled TTT transform of the data set in Figure 5.2. Since the empirical version of the scaled TTT transform is concave, it indicates that the hazard function is increasing.



Fig 5.2 The empirical scaled TTT transform of the data set

The empirical modelling involves the following steps:

1. Model description and estimation of its parameters;

2. Model validation; and

3. Model selection.

Effective modelling requires a good understanding of the properties of different models. The estimation of the parameter of the proposed model is obtained by the method of maximum likelihood (ML) estimation. To check the validity of the model, we compute the Kolmogorov-Smirnov (KS) distance between the empirical distribution function and the fitted distribution function when the parameters are obtained by the method of maximum likelihood. 
The following graphical methods are used for suitability of the model under consideration:

a) Quantile-Quantile (QQ) plot and

b) Probability -Probability (PP) plot.

\section{Computation of MLE}

The maximum likelihood estimates (MLEs) are obtained by direct maximization of the loglikelihood function $\ell(\alpha, \lambda, \theta)$ given in (3.1). The advantage of this procedure is that it runs immediately using existing statistical packages such as R, [R Development Core Team, (2019)]. We consider the software $\mathrm{R}$ through the QuasiNewton algorithm Lange (1999) to compute the MLEs.

Table 5.2 shows the ML estimates, standard error (SE) and $95 \%$ confidence intervals for parameters alpha, lambda and theta. The maximized value of loglikelihood is $\ell(\hat{\alpha}, \hat{\lambda}, \hat{\theta})=-141.885$.

Table 5.2 MLE, standard error and $95 \%$ confidence interval

\begin{tabular}{cccc}
\hline Parameter & MLE & Std. Error & 95\% Confidence Interval \\
\hline Alpha & 0.0678 & 0.00616 & $(0.0557,0.0799)$ \\
Lambda & 44.3476 & 3.9874 & $(36.5324,52.1628)$ \\
Theta & 2.5225 & 0.1719 & $(2.1856,2.8594)$ \\
\hline
\end{tabular}

\section{Model validation}

To check the validity of the model we compute the Kolmogorov-Smirnov (KS) distance between the empirical distribution function and the fitted distribution function when the parameters are obtained by the method of maximum likelihood is 0.0637 and the corresponding $p$-value is $=0.8117$. We have plotted the empirical distribution function and the fitted distribution function in Figure 5.3. From Figure 5.3, it is clear that the fitted Gompertz distribution provides good fit to the given data.

Further validation of this finding can be obtained by inspecting the probabilityprobability $(\mathrm{P}-\mathrm{P})$ and quantile-quantile (Q-Q) plots. A P-P plot depicts the points:

$\left(F\left(x_{(i)}\right), F\left(x_{(i)} ; \underline{\hat{\delta}}\right)\right) ; i=1,2, \ldots, n$

where $\hat{\hat{\delta}}=(\hat{\alpha}, \hat{\lambda}, \hat{\theta})$ and $x_{(i)}$ are the order statistics,

A Q-Q plot depicts the points:

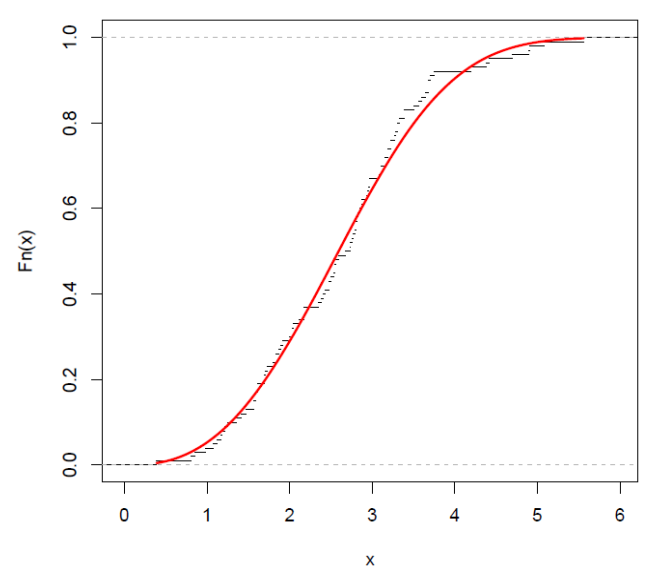

Fig 5.3 The graph of the empirical and fitted distribution function.

$$
\left(x_{(i)}, F^{-1}\left(\frac{i}{n+1} ; \underline{\hat{\delta}}\right)\right) ; i=1,2, \ldots, n
$$



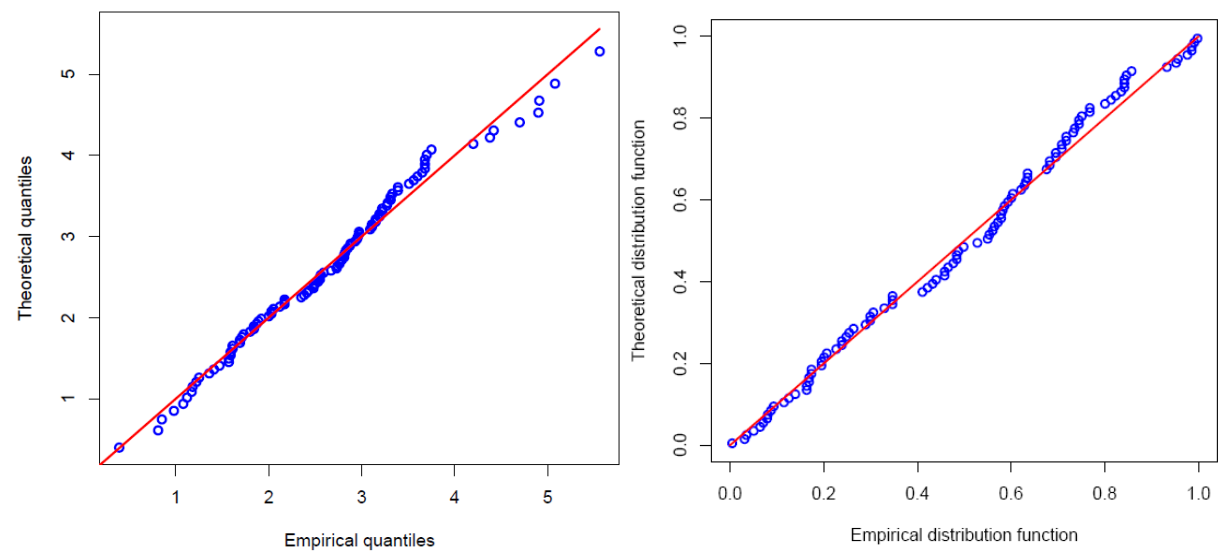

Fig 5.4O-O nlot usingMIFs as estimate Fig 5.5 P-P nlot using MIFs as estimate.

The Q-Q and P-P plots for the fitted model are shown in Figs. 5.4 and 5.5. It is evident that the fit of the Gompertz extension distribution is good.

\section{Bayesian analysis}

\section{OpenBUGS script for the Bayesian analysis of}

\section{Gompertz extension model}

model

\{

for $(\mathrm{i}$ in $1: \mathrm{N})$

\{

$\mathrm{x}[\mathrm{i}] \sim$ dgpz.ext_T(alpha, lambda, theta)

reliability[i] <- $(x$ [i], $x[i])$ \# to estimate reliability

$\mathrm{f}[\mathrm{i}]<-\operatorname{density}(\mathrm{x}[\mathrm{i}], \mathrm{x}[\mathrm{i}])$ \# to estimate density

$\operatorname{hrf[i]}<-\operatorname{hrf}(x[i], x[i]) \quad$ \# to estimate hazard

\# To predict the data set

$\mathrm{ep}[\mathrm{i}]<-(\mathrm{i}-0.5) / \mathrm{N}$

x.new $[\mathrm{i}]<-(1.0 /$ alpha $) * \log (1.0+\operatorname{pow}((1 /$ lambda $)$

$* \log (1.0-\mathrm{ep}[\mathrm{i}]), 1.0 /$ theta $))$

\}

\# Prior distributions of the model parameters

Alpha $\sim \operatorname{dunif}(0,10)$

lambda $\sim$ dgamma $(1,0.01)$

theta $\sim \operatorname{dgamma}(0.001,0.001)$

\}

\section{Data}

$\overline{\operatorname{list}(\mathrm{N}}=100, \mathrm{x}=\mathrm{c}(0.39,0.81,0.85,0.98,1.08,1.12$, $1.17,1.18,1.22,1.25,1.36,1.41,1.47,1.57,1.57$, $1.59,1.59,1.61,1.61,1.69,1.69,1.71,1.73,1.80$, $1.84,1.84,1.87,1.89,1.92,2.00,2.03,2.03,2.05$, $2.12,2.17,2.17,2.17,2.35,2.38,2.41,2.43,2.48$, $2.48,2.50,2.53,2.55,2.55,2.56,2.59,2.67,2.73,2.74$, $2.76,2.77,2.79,2.81,2.81,2.82,2.83$,
$2.85,2.87,2.88,2.93,2.95,2.96,2.97,2.97,3.09$, $3.11,3.11,3.15,3.15,3.19,3.19,3.22,3.22,3.27$, $3.28,3.31,3.31,3.33,3.39,3.39,3.51,3.56,3.60$, $3.65,3.68,3.68,3.68,3.70,3.75,4.20,4.38,4.42$, $4.70,4.90,4.91,5.08,5.56)$ )

\section{Initial values}

list(alpha $=0.01$, lambda $=5.0$, theta $=1.0) \#$ Chain 1 list (alpha $=0.1$, lambda $=10$., theta $=5.0) \quad \#$ Chain2

We assume the independent uniform prior for $\alpha \sim U\left(a_{1}, b_{1}\right)$ and gamma priors for $\lambda \sim G\left(a_{2}, b_{2}\right) \quad$ and $\theta \sim G\left(a_{3}, b_{3}\right)$ with hyperparameter values $a_{1}=0, b_{1}=10.0$, $a_{2}=1.0, b_{2}=0.01, a_{3}=0.001, b_{3}=0.001$.

We run the model to generate two Markov Chains at the length of 40,000 with different starting points of the parameters.

We have chosen initial values $(\alpha=0.01, \lambda=5.0, \theta=1.0)$ for the first chain and ( $\alpha=0.1, \lambda=10.0, \theta=5.0)$ the second chain. The convergence is monitored using trace and ergodic mean plots; we find that the Markov Chain converges together after approximately 2000 observations. Therefore, the burning of 5000 samples is more than enough to erase the effect of the starting point (initial values). Finally, samples of size 7000 are formed from the posterior by picking up equally spaced every fifth outcome (to minimize the autocorrelation 
among the generated deviates.), i.e. thin $=5$, starting from 5001.

Therefore, we have the posterior samples from chain 1 and chain 2 as

$\left(\alpha_{1}^{(j)}, \lambda_{1}^{(j)}, \theta_{1}^{(j)}\right) ; j=1, \ldots, 7000$

and $\left(\alpha_{2}^{(j)}, \lambda_{2}^{(j)}, \theta_{2}^{(j)}\right) ; j=1, \ldots, 7000$.

Chain 1 is considered for convergence diagnostics plots. The visual summary is based on a posterior sample obtained from chain 1, whereas the numerical summary is presented for both the chains.

\section{Convergence diagnostics}

Before examining the parameter estimates or performing other inference, it is a good idea to look at plots of the sequential(dependent) realizations of the parameter estimates and plots thereof. The sequential plot of parameters is the plot that most often exhibits difficulties in the Markov chain. Figure 5.6 shows the sequential realizations of the parameters of the model.

\section{History (Trace) Plot:}
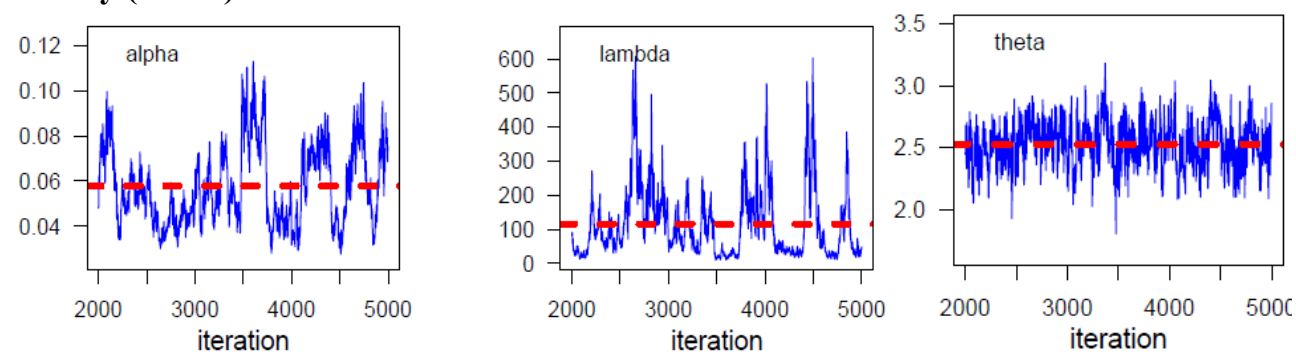

Fig 5.6 Sequential realization of the parameters $\alpha, \lambda$ and $\theta$.

It looks like nice oscillograms around a horizontal line without any trend. The Markov chain is most likely to be sampling from the stationary distribution and is mixing well.

\section{Running Mean (Ergodic mean) Plot:}
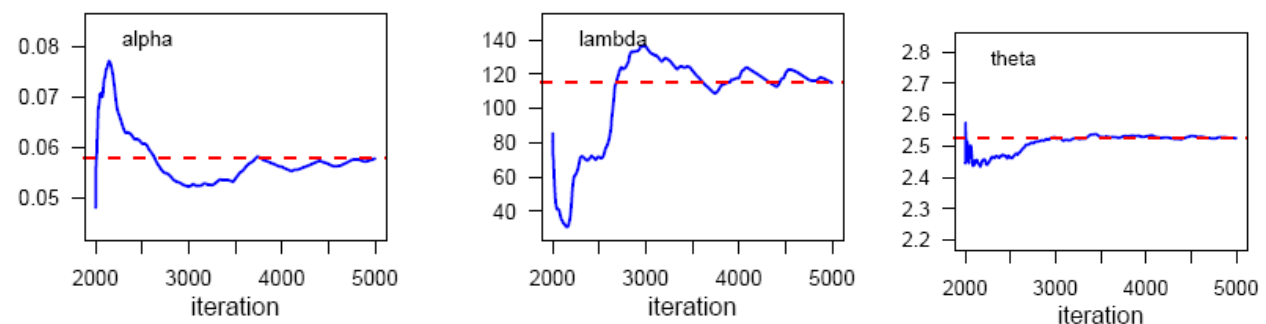

Fig.5.7 The Ergodic mean plots for $\alpha, \lambda$ and $\theta$.

Generate a time series (iteration number) plot of the running mean for each parameter in the chain. The running mean is computed as the mean of all sampled values up to and including that at aiven iteration. The convergence pattern based on ergodic averages is shown in Figure 5.7, indicating the convergence of the chain. 


\section{Autocorrelation:}

The graph shows that the correlation is almost negligible. We may consider an independent sample from the target distribution, i.e. posterior.
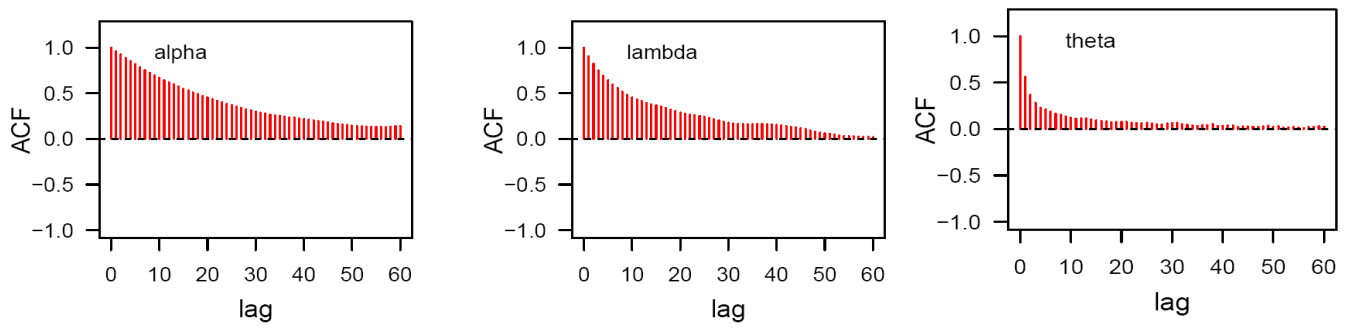

\section{Brooks-Gelman-Rubin (BGR) diagnostic:}

The Brooks, Gelman and Rubin convergence diagnostic is appropriate for the analysis of two or more parallel chains, each with different starting values which are overdispersed to the target distribution. The green line represents the between variability, the blue line represents the within variability, and the red line represents the ratio. Evidence for convergence comes from the red line being close to 1 From Figure 5.9; it is clear that convergence is achieved. Thus, we can obtain the posterior summary statistics.

$\mathrm{On}$
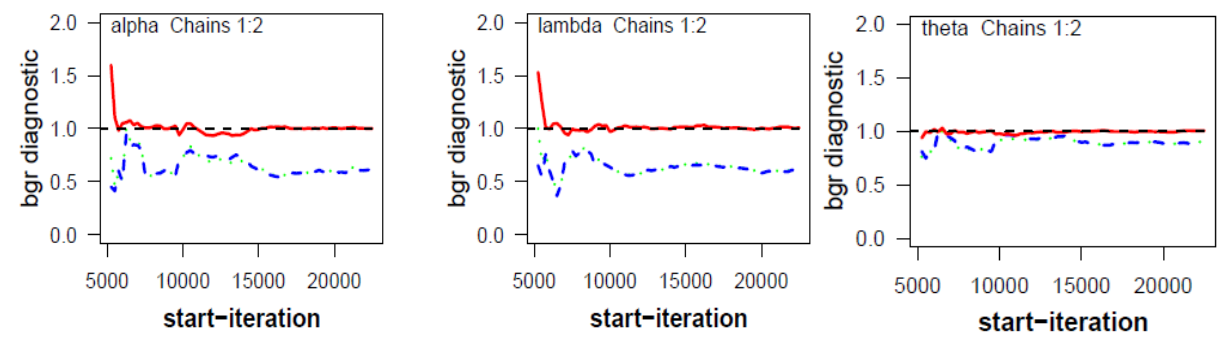

\section{Posterior Analysis:}

\section{(a). Numerical Summary}

The numerical summary is presented $\left(\alpha_{1}^{(j)}, \lambda_{1}^{(j)}, \theta_{1}^{(j)}\right) ; j=1, \ldots, 7000$ from chain 1 and $\left(\alpha_{2}^{(j)}, \lambda_{2}^{(j)}, \theta_{2}^{(j)}\right) ; j=1, \ldots, 7000$ chain 2 .

We have considered various quantities of interest and their numerical values based on the MCMC sample of posterior characteristics for Gompertz extension distribution. The MCMC results of the posterior mean, mode, standard deviation (SD), first quartile, median, third quartile, $2.5^{\text {th }}$ percentile, $97.5^{\text {th }}$ percentile, skewness, arepresented in Table 5.3 for both the chains. The 95\% symmetric and HPD credible intervals of the parameters $\alpha \lambda$, and $\theta$ are displayed in Table 5.3 based on posterior samples from chain 1 only.

The advantage of using the MCMC method over the MLE method is that we can always obtain a reasonable interval estimate of the parameters by constructing the probability intervals based on the empirical posterior distribution. This is often unavailable in maximum likelihood estimation. The algorithm described by Chen and Shao (1999), is used to compute the HPD intervals under the assumption of the unimodal marginal posterior distribution. The width of the HPD is another way of measuring uncertainty of beliefs. If the HPD is wide, then beliefs are uncertain. If the HPD is narrow, then beliefs are fairly certain. 
Table 5.3: Numerical summaries based on MCMC sample of posterior characteristics for Gompertz extension distribution

\begin{tabular}{lcccccc}
\hline \multicolumn{1}{c}{ Characteristics } & alpha & $\begin{array}{c}\text { Chain 1 } \\
\text { lambda }\end{array}$ & theta & alpha & $\begin{array}{c}\text { Chain 2 } \\
\text { lambda }\end{array}$ & theta \\
\hline Mean & 0.0691 & 82.162 & 2.5157 & 0.0727 & 90.883 & 2.500 \\
Standard Deviation & 0.0279 & 40.669 & 0.2037 & 0.0415 & 96.309 & 0.218 \\
Minimum & 0.0204 & 0.577 & 1.5920 & 0.0206 & 0.183 & 1.495 \\
2.5th Percentile $\left(\mathrm{P}_{2.5)}\right)$ & 0.0339 & 4.043 & 2.1150 & 0.0326 & 2.393 & 2.046 \\
First Quartile $\left(\mathrm{Q}_{1}\right)$ & 0.0503 & 25.090 & 2.3800 & 0.0478 & 22.243 & 2.372 \\
Median & 0.0628 & 57.635 & 2.5150 & 0.0623 & 57.380 & 2.508 \\
Third Quartile $\left(\mathrm{Q}_{3}\right)$ & 0.0811 & 112.10 & 2.6530 & 0.0846 & 129.52 & 2.646 \\
97.5th Percentile( $\left.\mathrm{P}_{97.5}\right)$ & 0.1454 & 306.16 & 2.9120 & 0.1711 & 350.02 & 2.892 \\
Maximum & 0.2821 & 443.90 & 3.2910 & 0.4402 & 519.00 & 3.296 \\
Mode & 0.0536 & 31.022 & 2.4987 & 0.0489 & 18.97 & 2.499 \\
Skewness & 1.7491 & 2.046 & -0.0616 & 3.4189 & 2.010 & -0.526 \\
\hline
\end{tabular}

Table 5.4 : 95\% symmetric and HPD credible intervals

\begin{tabular}{ccc}
\hline Parameter & Credible Interval & HPD Credible Interval \\
\hline alpha & $(0.03391,0.1454)$ & $(0.02682,0.1231)$ \\
lambda & $(4.0429,306.16)$ & $(0.5765,244.3)$
\end{tabular}

\section{(b) Visual Summary}

The visual graphs include the boxplot, density strip plot, histogram, marginal posterior density estimate and rug plots for the parameters. We have also superimposed the 95\% HPD intervals.
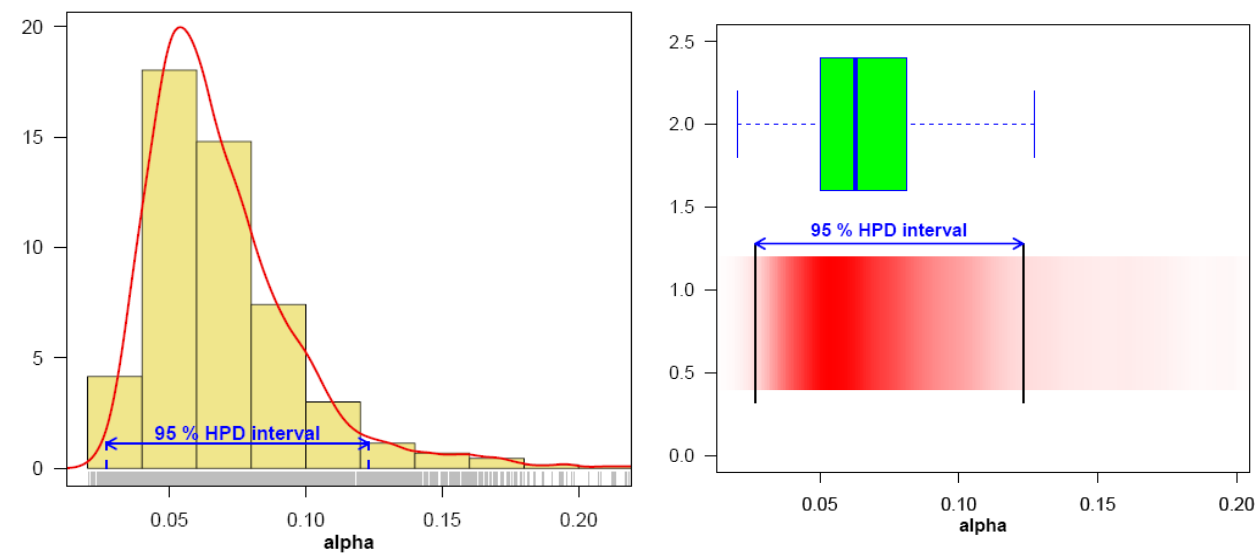

Fig 5.10Left panel: Histogram, marginal posterior density and 95\% HPD interval ; Right panel: boxplot and density strip of $\alpha$, based on the posterior sample.

These graphs provide an almost complete picture of the posterior uncertainty about the parameters. We have used the posterior sample $\left(\alpha_{1}^{(j)}, \lambda_{1}^{(j)}, \theta_{1}^{(j)}\right) ; j=1, \ldots, 7000$ to draw these graphs.

The density strip illustrates a univariate distribution as a shaded rectangular strip, whose darkness at a point is proportional to the probability density. The strip is darkest at the maximum density and fades 
into the background at the minimum density. It may be used to generalise the common point and line drawing of a point and interval estimate, by representing the entire posterior or predictive distribution of the estimate, Jackson (2008).

Histograms can provide insights on skewness, behaviour in the tails, presence of multi-modal behaviour, and data outliers; histograms can be compared to the fundamental shapes associated with standard analytic distributions.
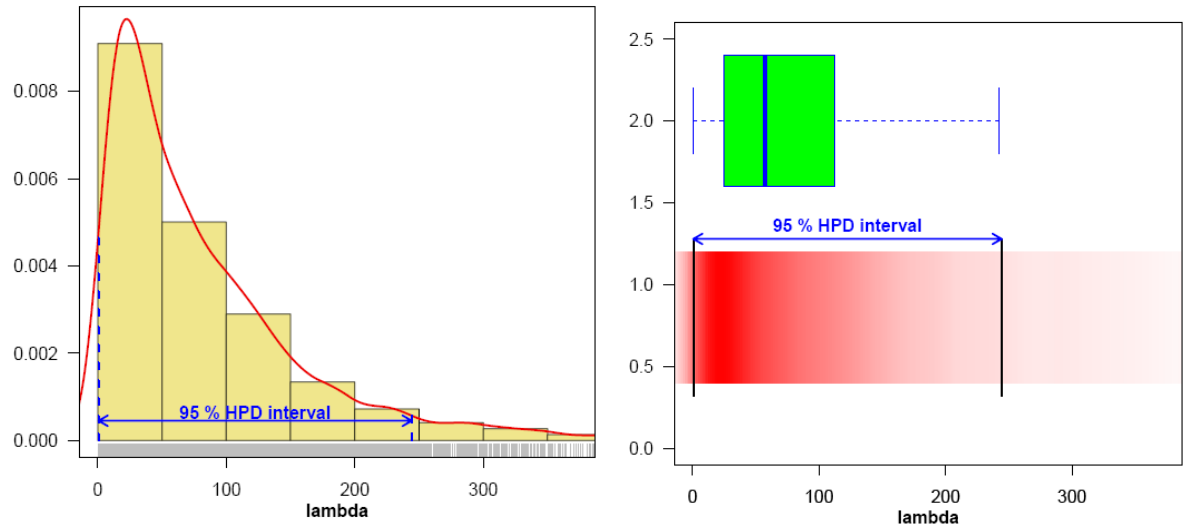

Fig 5.11Left panel: Histogram, marinal posterior density and 95\% HPD interval ;

Right panel: boxplot and density strip of $\lambda$, based on a posterior samples.

Figure 5.10 represents the histogram, marginal posterior density and 95\% HPD interval for $\alpha$ (left panel) and boxplot and density strip plot (right panel). We have plotted similar graphs for $\lambda \theta$ and displayed in Figure 5.11 and 5.12. The kernel density estimates have been drawn using $\mathrm{R}$ with the assumption of Gaussian kernel and properly chosen values of the bandwidths. It can be seen that $\theta$ is symmetric whereas $\alpha, \lambda$ and show positive skewness.
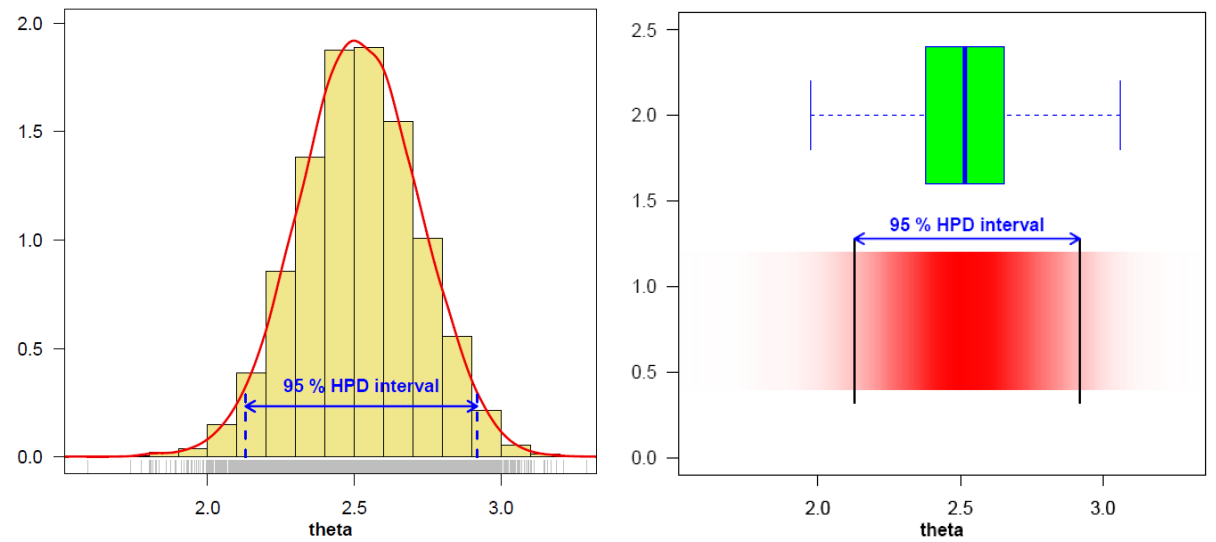

Fig 5.12Left panel : Histogram, marinal posterior density and 95\% HPD interval ;

Right panel: boxplot and density strip of $\theta$, based on a posterior sample.

\section{Comparison with MLE}

We have used a graphical method for the comparison of Bayes estimates with ML estimates. In Figure 5.13 , the density functions $f(x ; \hat{\alpha}, \hat{\lambda}, \hat{\theta})$ using MLEs and Bayesian estimates (the posterior means), computed via MCMC samples, are plotted. It is evident from Figure 5.13 that the MLEs and the Bayes estimates are quite close and fit the data very well. 
Further support for this finding can be obtained by inspecting Figure 5.14. In Figure 5.14, we have plotted $2.5^{\text {th }}, 50^{\text {th }}$ and $97.5^{\text {th }}$ quantiles of the estimated density; it can be considered as an evaluation of model fit, based on the posterior sample, $\left(\alpha_{1}^{(j)}, \lambda_{1}^{(j)}, \theta_{1}^{(j)}\right) ; j=1, \ldots, 7000$.

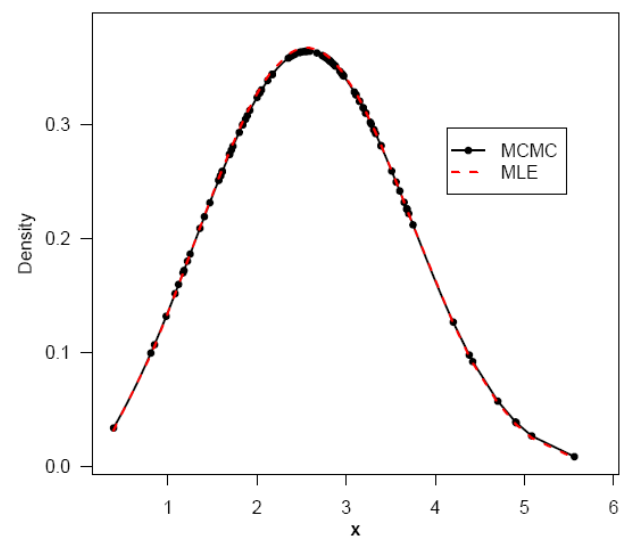

Fig 5.13 The density functions using ML

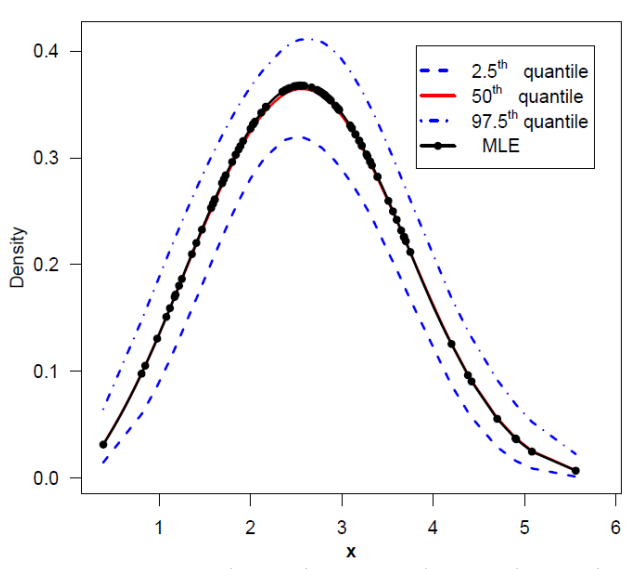

Fig 5.14 Density estimatesand Bayesian estimates

We have computed the density function at each observed data point for 7000 posterior samples, using logical function density () in OpenBUGS

$f\left(x_{i} ; \alpha_{1}^{(j)}, \lambda_{1}^{(j)}, \theta_{1}^{(j)}\right) ; j=1, \ldots, 7000 ; i=1, \ldots, 100$.

The density corresponding to MLE has been plotted using the "plug-in" estimates of the parameters. It shows that we have a fairly good model for the given data set.

\section{Estimation of the reliability function}

In this section, our main aim is to demonstrate the effectiveness of the proposed methodology. For this, we have estimated the reliability function using posterior samples. Since we have an effective MCMC technique, we can estimate any function of the parameters.

We have used the Kaplan-Meier estimate of the reliability function to make the comparison more meaningful. The Figure 5.15, exhibits the estimated reliability function (dashed blue line: $2.5^{\text {th }}$ and $97.5^{\text {th }}$ quantiles; solid red line: $50^{\text {th }}$ quantile) using Bayes estimate based on MCMC output and the empirical reliability function (solid black line). Figure 5.15 shows that reliability estimate based on MCMC is very close to the empirical reliability estimates.

\section{Estimation of hazard and reliability at} $X_{(30)}: t=2.0$

Indeed, the MCMC samples may be used to summarize the posterior uncertainty about the parameters $\alpha$ completely, $\lambda$ and $\theta$ through a

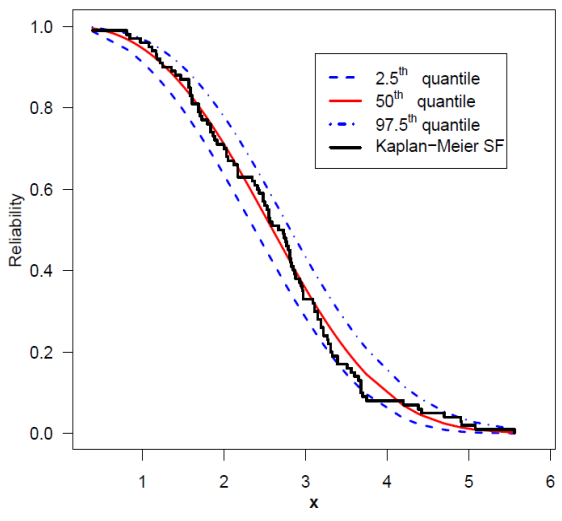

Fig 5.15 Reliability function estimate using kernel estimate of the posterior distribution. 
This is also true of any function of the parameters, e.g. reliability and hazard functions. Suppose we wish to give point and interval estimates for reliability and hazard functions at the mission time $t=2.0$ (at the $30^{\text {th }}$ observed data point).

We have computed the hazard and reliability functions at mission time $t=2.0$ (at the $30^{\text {th }}$ observed data point) for 7000 posterior samples, using logical function $h r f()$ and reliability (), Kumar, et al., (2010), in OpenBUGS. It can be computed directly using hazard and reliability functions given in (4.1.4) and (4.1.3), respectively.
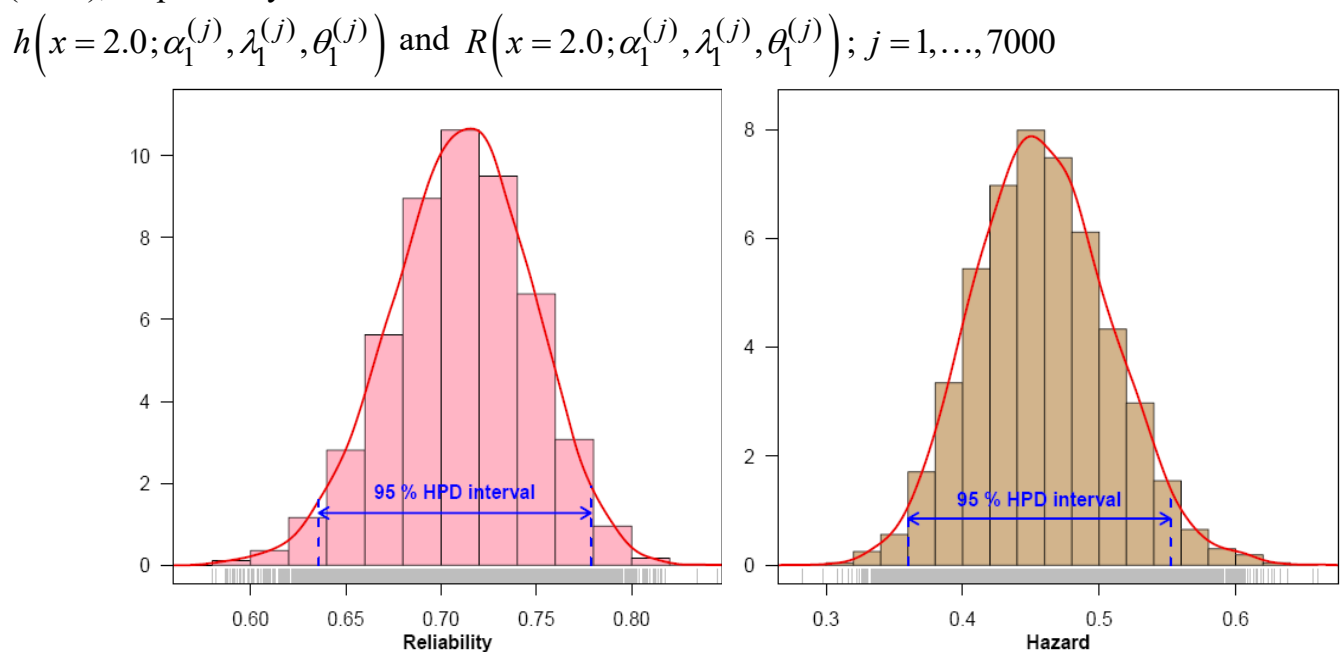

Fig. 5.16 Visual summary of reliability(left panel) and hazard(right panel) at $t=2.0$

The marginal posterior density estimates of the reliability (left panel) and hazard functions(right panel) and their histograms based on samples of size 7000 are shown in Figure. 5.16 using the Gaussian kernel. It is evident from the estimates that the marginal distribution of reliability is negatively skewed, whereas hazard is positively skewed.
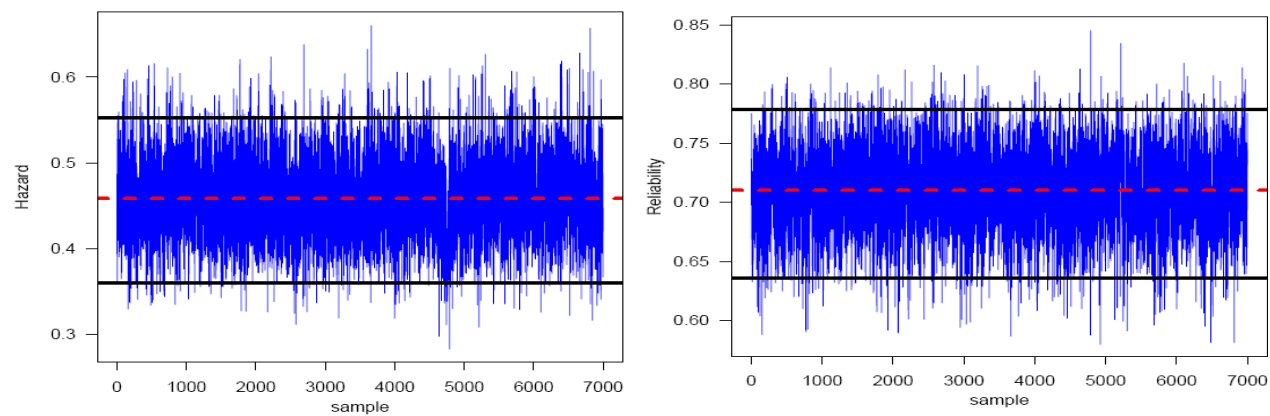

Fig 5.17 MCMC output of $h(t=2.0)$ and $R(t=2.0)$. Dashed line(...) represents the posterior median and solid lines (-) represent lower and upper bounds of $90 \%$ probability intervals (HPD).

A trace plot is a plot of the iteration number against the value of the draw of the parameter at each iteration. Figure 5.17 displays 7000 chain values for the hazard $h(t=2.0)$ and reliability $R(t=2.0)$ functions, with their sample median and $90 \%$ credible intervals. The MCMC results of the posterior mean, mode, standard deviation (SD), five-point summary statistics (minimum, first quartile, median, third quartile and maximum), $2.5^{\text {th }}$ percentile, $97.5^{\text {th }}$ percentile, skewness, 95\% symmetric and HPD credible intervals of reliability and hazard functions are displayed in Table 5.5. 
Nepal Journal of Science and Technology (NJST) (2020), 19(1)

Table 5.5 Posterior summary for reliability and hazard at $t=2.0$

\begin{tabular}{lcc}
\hline \multicolumn{1}{c}{ Characteristics } & Reliability & Hazard \\
Mean & 0.7103 & 0.4584 \\
Standard Deviation & 0.0367 & 0.0499 \\
Minimum & 0.5798 & 0.2830 \\
2.5th Percentile $\left(\mathrm{P}_{2.5}\right)$ & 0.6358 & 0.3658 \\
First Quartile $\left(\mathrm{Q}_{1}\right)$ & 0.6863 & 0.4238 \\
Median & 0.7114 & 0.4564 \\
Third Quartile $\left(\mathrm{Q}_{3}\right)$ & 0.7355 & 0.4910 \\
97.5th Percentile $\left(\mathrm{P}_{97.5}\right)$ & 0.7786 & 0.5589 \\
Maximum & 0.8452 & 0.6599 \\
Mode & 0.7169 & 0.4495 \\
Skewness & -0.1692 & 0.1913 \\
95\% Credible Interval & $(0.6358,0.7786)$ & $(0.3658,0.5589)$ \\
95\% HPD Credible Interval & $(0.6358,0.7786)$ & $(0.3602,0.5521)$ \\
\hline
\end{tabular}

The ML estimates of reliability and hazard function at $t=2.0$ are computed using the invariance property of the MLE. The ML estimates of hazard and reliability are $\hat{h}(t=2.0)=0.4606$ and $\hat{R}(t=2.0)=0.7170$ respectively.

\section{Model compatibility:}

\section{Posterior predictive checks}

A natural way to assess the fit of a Bayesian model is to look at how well the predictions from the model agree with the observed data, (Gelman 2003); (Gelman et al., 2004). We do this by comparing the posterior predictive simulations with the data. 

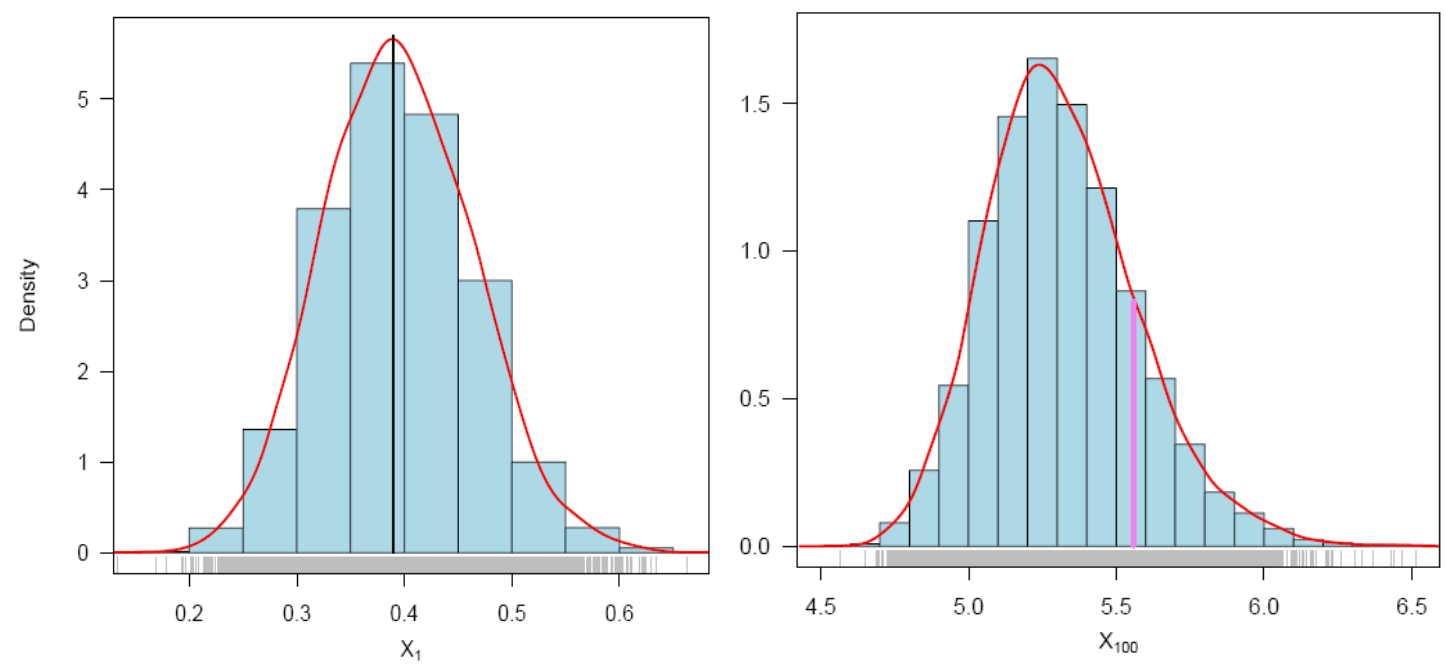

Fig 5.18 Density estimates of the smallest $\left(\mathrm{X}_{(1)}\right)$ and largest $\left(\mathrm{X}_{(100)}\right)$ order future observations, vertical linesrepresent corresponding observed values

There are several approaches available for the study of model compatibility in the Bayesian framework. Predictive simulation is an easiest and flexible one. The basic idea of studying the model compatibility through predictive simulation is to compare the observed data or some function of it with the data that would have been anticipated from the assumed model called the predictive data. If the two data sets compare favourably, the assumed model can be considered to be an appropriate choice for the data in hand (Gupta, et al., 2008).

Modern Bayesian computational tools, however, provide straightforward solutions as one can easily simulate predictive samples if MCMC outputs are available from the posterior corresponding to the assumed model. Most of the standard numerical and graphical methods based on predictive distribution can then be easily implemented to study the compatibility of the model. One of the best ways to assess model adequacy based on posterior predictive

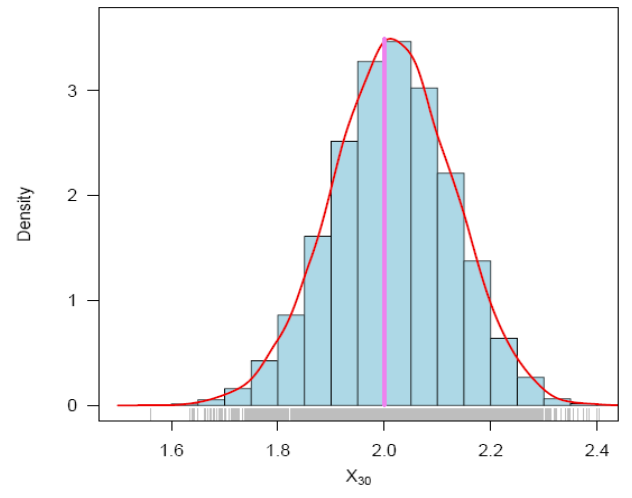

Fig 5.19 Density estimates of the $X_{(30)}$, vertical lines represent corresponding observed values distributions is graphical.

To obtain further clarity on our conclusion for the study of model compatibility, we have considered plotting of density estimates of smallest, largest and $30^{\text {th }}$ smallest, i.e. $\left(X_{(1)}, X_{(100)}\right.$ and $\left.X_{(30)}\right)$ replicated future observations from the model with the superimposed corresponding observed data. For this purpose, 2000 samples have been drawn from the posterior using MCMC procedure and then

The density estimates based on replicated future data sets are shown in Figures 5.18 and 5.19. Figure 5.18 represents the estimates corresponding to the smallest and largest predictive observations, whereas the same for $30^{\text {th }}$ smallest observations is shown in Figure 5.19. The corresponding observed values are also shown using vertical lines. obtained predictive samples from the model under consideration using each simulated posterior sample. The size of predictive samples is the same as that of the observed data. To 
obtain further clarity on our conclusion for the study of model compatibility, we have considered plotting of density estimates of smallest, largest and $30^{\text {th }}$ smallest, i.e. $\left(X_{(1)}, X_{(100)}\right.$ and $\left.X_{(30)}\right)$ replicated future observations from the model with the superimposed corresponding observed data. For this purpose, 2000 samples have been drawn from the posterior using MCMC procedure and then obtained predictive samples from the model under consideration using each simulated posterior sample. The size of predictive samples is the same as that of the observed data.

The MCMC results of the posterior mean, median, mode of smallest and largest $\left(\mathrm{X}_{(1)}\right.$ and $\left.\mathrm{X}_{(100)}\right)$ and $\mathrm{X}_{(30)}$ are displayed in Table 5.6.

Table 5.6: Posterior characteristics

\begin{tabular}{lllll}
\hline & Observed & Mode & Mean & Median \\
\hline $\mathbf{X}_{(\mathbf{1})}$ & 0.39 & 0.388 & 0.395 & 0.441 \\
$\mathbf{X}_{(\mathbf{3 0 )}}$ & 2.00 & 2.009 & 2.015 & 2.092 \\
$\mathbf{X}_{(\mathbf{1 0 0})}$ & 5.56 & 5.234 & 5.316 & 5.473 \\
\hline
\end{tabular}

In fact, we have predicated the entire data set. Figure 5.20 represents the Q-Q plot of predicted quantiles vs observed quantiles. We, therefore, conclude that the Gompertz extension model is compatible with the given data set.

As Figures 5.18 and 5.19 show, the posterior predictive distributions are centred over the observed values, which indicates a good fit. In general, the distribution of replicated data appearsto match that of the observed data fairly well. Overall, the results of the posterior predictive simulation indicate that the model fits these data particularly well.

\section{Conclusion}

We have discussed the Markov chain Monte Carlo (MCMC) method to compute the Bayesian estimates of the parameters, hazard and reliability functions of

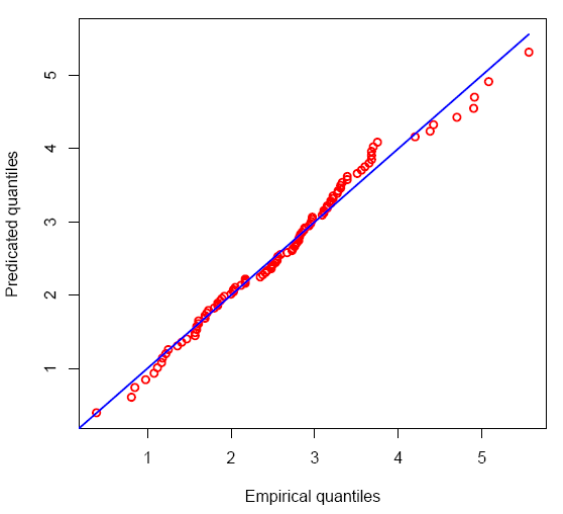

Fig. 5.22 Q-Q plot of predictive quantiles versus empirical quantiles Gompertz extension distribution based on a complete sample. We have obtained the probability intervals for parameters, hazard and reliability functions. We have presented the model compatibility via the posterior predictive check method. We have applied the developed techniques on a real data set. Thus, the tools developed can be applied for full Bayesian analysis of Gompertz extension model. 


\section{References}

Aarset, M.V. (1987). How to identify a bathtub hazard function. IEEE Transactions on Reliability. 36. 106-108.

Alizadeh, M., Afify, A.Z., Eliwa, M.S., \& Ali, S.(2020). The odd log-logistic Lindley-G family of distributions: properties, Bayesian and nonBayesian estimation with applications. Comput Stat 35, 281-308.

Afify, A.Z., Suzuki, A.K., Zhang, C. \& Nassar, M. (2019). On three-parameter exponential distribution: properties, Bayesian and nonBayesian estimation based on complete and censored samples, Communications in Statistics Simulation and Computation, DOI: 10.1080/03610918.2019.1636995

Chen, M. H. \& Shao, Q. M. (1999). Monte Carlo estimation of Credible Bayesian intervals and HPD intervals. Journal of Computational and Graphical Statistics. 8(1). 69-92.

Chen, Z. (2000). A new two-parameter lifetime distribution with bathtub shape or increasing failure rate function. Statistics and Probability Letters. 49. 155-161.

Dhillon, B.S. (1981). Life Distributions. IEEE Transactions on Reliability. 30. 457-459.

Gelfand, A.E. \& Smith, A.F.M.(1990). Samplingbased approach to calculating marginal densities. Journal of the American Statistical Association. 85. 398-409.

Gelman, A. (2003). A Bayesian Formulation of Exploratory Data Analysis and Goodness-of-fit Testing. International Statistical Review.71(2). 369-382.

Gelman, A., Carlin, J., Stern, H., \& Rubin, D. (2004). Bayesian Data Analysis. Second Edition. London, Chapman \& Hall.

Geman, S.\& Geman, D. (1984). Stochastic Relaxation. Gibbs Distributions and the Bayesian Restoration of Images. IEEE Transactions on Pattern Analysis and Machine Intelligence. 6. 721-741.

Gompertz, B. (1825). On the nature of the function expressive on the law of human mortality. Philosophical transactions of the Royal society of London, 115. 513-583.

Gupta, A., Mukherjee, B. \& Upadhyay, S.K. (2008). A Bayes study using Markov Chain Monte Carlo simulation. Reliability Engineering \& System Safety. 93. 1434-1443.

Hastings, W.K.(1970). Monte Carlo sampling methods using Markov chains and their applications. Biometrika. 57. $97-109$.
Jackson, C.H. (2008). Displaying uncertainty with shading. The American Statistician. 62 (4). 340347.

Kumar, V. (2010). Bayesian analysis of exponential extension model, J. Nat. Acad. Math., 24. 109-128.

Kunitz, H. (1989). A new class of bathtub-shaped hazard rates and its application in a comparison of two test-statistics. IEEE Transactions on Reliability. 38. 351-354.

Lange, K. (1999). Numerical Analysis for Statisticians. Springer, New York, NY.

Leemis, L. M. (1986). Lifetime distribution identities. IEEE Transactions on Reliability. 35. 170-174.

Lunn, D.(2010). Recent Developments in the BUGS software. ISBA Bulletin. 17(3). 16-17.

Lunn, D.J., Jackson, C., Best, N., Andrew, A.\& Spiegelhalter, D. (2013). The BUGS Book: A Practical Introduction to Bayesian Analysis. Chapman \& Hall/CRC, London, UK.

Marshall, A. W., \& Olkin, I. (1997). A new method for adding a parameter to a family of distributions with application to the exponential and Weibull families. Biometrika 84 (3). 641-652.

Marshall, A. W., \& Olkin, I.(2007). Life Distributions: Structure of Nonparametric, Semiparametric, and Parametric Families. Springer, New York.

Metropolis, N., Rosenbluth, A.W., Rosenbluth, M.N., Teller, A.H. and Teller, E.(1953). Equations of state calculations by fast computing machines. Journal Chemical Physics. 21. 1087-1091.

Murthy, D.N.P., Xie, M. \& Jiang, R. (2004). Weibull Models, Wiley, New York.

Nichols, M.D. \& Padgett, W.J. (2006). A bootstrap control chart for Weibull percentiles. Quality and Reliability Engineering International. 22. 141-151.

R Development Core Team(2019). R:A language and environment for statistical computing. $\mathrm{R}$ Foundation for Statistical Computing, Vienna, Austria, ISBN 3-900051-07-0, URL http://www.Rproject.org.

Thomas, A.(2010). OpenBUGS Developer Manual, version 3.1.2. http://www.openbugs.info/

Thomas, A., O'Hara, B., Ligges, U. \& Sturtz, S. (2006). Making BUGS Open. R News. 6. 12-17, URL http://mathstat.helsinki.fi/openbugs/.

Xie, M., Tang, Y.\& Goh, T.N. (2002). A modified Weibull extension with bathtub-shaped failure rate function, Reliability Engineering \& System Safety, 76. $279-285$. 